\title{
Differences in the INR evaluation of two different thromboplastins in patients with positivity to lupus anticoagulant in ongoing oral anticoagulation
}

This article was published in the following Dove Press journal:

Journal of Blood Medicine

30 April 2010

Number of times this article has been viewed

\author{
Paola Ferrazzi ${ }^{1,3}$ \\ Anna Colombo' \\ Pierpaolo Di Micco',2 \\ Corrado Lodigiani' \\ Luca Librè' \\ Lidia Luciana Rota' \\ Alessandro Montanelli ${ }^{3}$ \\ Ilaria Quaglia' \\ 'Thrombosis Center, Istituto Clinico \\ Humanitas IRCCS, Rozzano (MI), Italy; \\ ${ }^{2}$ Internal Medicine, Fatebenefratelli \\ Hospital of Naples, Italy; ${ }^{3}$ Unit of \\ Laboratory and Clinical Chemistry, \\ Istituto Clinico Humanitas IRCCS, \\ Rozzano (MI), Italy
}

Background: A possible interference between lupus anticoagulant (LAC), a well characterized clotting inhibitor, in the International Normalized Ratio (INR) determination during oral anticoagulation (OA) has been reported in the literature. Few data are available about the relationship between this kind of interference and the daily clinical management of oral anticoagulation. The aim of the study is to evaluate the role of two different thromboplastins-RecombiPlasTin $2 \mathrm{G}$ and HepatoComplex-in the determination of INR values of several patients' ongoing OA for a previous thrombotic disorder with and without positivity to LAC, and to evaluate possible interferences in the daily therapeutic approach.

Patients and methods: We selected 16 patients (13 females and 3 males, mean age $59 \pm 16$ years) with LAC positivity ongoing OA and 11 control subjects ( 7 females and 4 males, mean age $58 \pm 14.5$ years) with similar characteristics (ie, ethnic background and weight) with LAC negativity ongoing OA. 165 assays for INR determination were analyzed from both groups. Statistical analysis was performed using STATA 10 software. $P$ values were considered significant if $<0.05$.

Results: Mean values of INR for patients with LAC positivity were $3.79 \pm 1.63$ when tested with RecombiPlasTin $2 \mathrm{G}$ vs $3.18 \pm 1.15$ when tested with HepatoComplex $(P<0.001$, s); while mean values of INR for patients with antiphospholipid syndrome (APS) with LAC negativity were $3.54 \pm 1.39$ when tested with RecombiPlasTin $2 \mathrm{G}$ vs $3.23 \pm 1.14$ when tested with HepatoComplex $(P<0.002$, s). An INR value $>$ than 4.5 was found in $31 / 165$ samples in 9 subjects, 8 patients with LAC positivity, and 1 control group subject with LAC negativity. There was a great difference in INR values in these subjects if we use the common thromboplastin (ie, RecombiPlasTin $2 \mathrm{G}$ ) with a INR range varying from $5.14 \pm 0.35$ vs $3.79 \pm 0.38$ if we use another thromboplastin (ie, HepatoComplex) $(P<0.001, \mathrm{~s})$. A change in the therapeutic approach for OA is possible in these cases because different INR values were obtained using different thromboplastins.

Discussion: Our data confirm that INR evaluation does not reveal significant changes also if tested with two different thromboplastins, for patients ongoing OA with and without LAC positivity, when the INR value is $<$ than 4 . Over this INR value there is a significant difference in patients with LAC positivity if we use a different thromboplastin for the INR determination. For this reason values obtained by RecombiPlasTin $2 \mathrm{G}$ need to be confirmed and matched with another thromboplastin (ie, HepatoComplex). This approach may be useful in order to have a good INR testing for the chronic long-term treatment with OA in particular in patients with LAC positivity.

Keywords: lupus anticoagulant, oral anticoagulation, INR, thromboplastin, prothrombin time, thrombosis, long-term treatment
Thrombosis Center, Istituto Clinico

Humanitas, Rozzano, Milan, Italy

$\mathrm{Tel}+390282244724$

Fax +390282 244724

Email paola.ferrazzi@humanitas.it 


\section{Introduction}

Oral anticoagulation (OA) is the gold standard therapy for long-term treatment of thrombotic disorders. ${ }^{1} \mathrm{OA}$ is based on the administration of antivitamin K (AVK) drugs that are able to induce a reduced intake of vitamin $\mathrm{K}$ and a following reduced synthesis of clotting factors (ie, clotting factors II, VII, IX, X). In particular, reductions of synthesized prothrombin (ie, clotting factor II), induces a prolonged prothrombin time (PT). ${ }^{2}$

PT is used in the daily clinical management of patients ongoing OA, and in the last years the International Normalized Ratio (INR) of PT is used to monitor patients ongoing OA. ${ }^{3}$ Moreover, OA monitoring is performed worldwide by INR. ${ }^{3}$

However, because of several reagents used to perform the PT INR, (ie, commercial thromboplastins), we sometimes observe differences in the INR evaluation. ${ }^{4}$

Lupus anticoagulant (LAC) is an acquired clotting inhibitor that is frequently associated with primary or secondary antiphospholipid syndrome (APS) and is able to alter mainly activated partial thromboplastin time (aPTT) and in few cases PT INR too. ${ }^{5}$ However, APS associated with LAC is frequently associated with thrombotic events and the long term antithrombotic treatment is usually performed by OA. ${ }^{6,7}$

Because the presence of LAC may alter also INR value during prolonged $\mathrm{OA}$, a misunderstanding in the daily clinical management of patients with positivity to LAC ongoing OA is possible because of this kind of interference. Moreover, in these cases, if we suppose an interference by LAC in the INR value, it is necessary to perform the INR assay with another thromboplastin less sensitive to an acquired inhibitor as LAC, ${ }^{8}$ as suggested several authors.

The aim of this study is to evaluate differences and effects of two different thromboplastins, to monitor INR during OA in patients with LAC positivity and previous thrombotic events, and to evaluate if there is a different clinical approach if this interference is present. Another aspect is related to the possible alteration induced by the interference on the doseresponse administration of AVK drugs that may interfere in the frail balance of OA management due to the the risk of thrombotic andlor hemorragic events.

\section{Patients and methods}

\section{Patients}

We selected 16 patients (13 females and 3 males, mean age $59 \pm 6$ years) with LAC positivity after a thrombotic episode and 11 patients ( 7 females and 4 males $58 \pm 14.5$ years) with LAC negativity after a thrombotic event (ie, venous thromboembolism, ischemic stroke, acute coronary syndrome), as a control group.

All patients were ongoing OA and we analyzed INR samples from all patients of both groups with two different thromboplastin (ie, RecombiPlasTin 2G, common thromboplastin, and HepatoComplex, a less sensitive thromboplastin to acquired inhibitors). We performed this observational analysis for 6 months.

Each patient performed nearly 6.1 blood samples to monitor INR during OA for a total of 165 samples in the study population.

As control group we selected 11 subjects with similar characteristics (age, ethnic background, weight) ongoing OA but with LAC negativity.

Each control subject performed nearly 5.6 blood samples in the observational period.

We do not divide patients into INR range groups because this kind of division had been performed in other reports. ${ }^{8}$

\section{Methods}

Venous blood samples were taken from antecubital vein and put in a tube with sodium citrate $1 / 9 \mathrm{v} / \mathrm{v}$. All selected subjects in the study performed consecutive blood samples for the evaluation of INR for OA with two different commercial thromboplastins, ie, RecombiPlasTin $2 \mathrm{G}$ (Instrumentation Laboratory, Milan, Italy) and HepatoComplex IL (Instrumention Laboratory). INR evaluation was performed with coagulometer ACL TOP (Instrumentation Laboratory).

LAC testing was performed according to international guidelines and with common commercial kits. ${ }^{9}$ LAC determination was performed at the time of diagnosis and after 12 weeks according to the international guidelines; further determinations are considered after 6-12 months andlor after clinical resolution of thrombotic disorders.

\section{Statistical analysis}

Data were described as number and percentage or mean and standard deviation where appropriate. Differences were explored with Mann-Whitney $u$ test and Student's $t$-test, where appropriate. $P$ values were considered significant if less than 0.05. Analysis was performed with STATA 10 (Stata Corp, College Station, TX).

\section{Results}

Mean values of INR for patients with LAC positivity were $3.79 \pm 1.63$ when tested with RecombiPlasTin $2 \mathrm{G}$ vs $3.18 \pm 1.15$ when tested with HepatoComplex 
$(P<0.001, \mathrm{~s})$, while mean values of INR for patients with LAC negativity were $3.54 \pm 1.39$ when tested with RecombiPlasTin $2 \mathrm{G}$ vs $3.30 \pm 1.07$ when tested with HepatoComplex $(P<0.002, \mathrm{~s})$.

INR value $>$ than 4.0 , so suggesting an overdose of AVK drugs, was found in 31/165 samples by 9 subjects, 8 patients with LAC positivity, and 1 control group subject with LAC negativity.

The mean INR values of patients with LAC positivity were $5.14 \pm 0.35$ when tested with RecombiPlasTin vs $3.79 \pm 0.38$ when tested with HepatoComplex $(P<0.001$, s), so confirming an interference of the acquired inhibitor LAC in the INR determination, which may influence the administered doses of AVK drugs in the daily therapeutic approach to OA.

Concerning the AVK doses, patients with LAC positivity had weekly doses of $28.55 \mathrm{mg}$ of warfarin vs $27.8 \mathrm{mg}$ for LAC negativity ( $P 0.48$, ns). Data are reported as cases vs controls linear regression in Figure 1.

\section{Discussion}

$\mathrm{OA}$ is the gold standard long-term treatment for thrombotic disorders also if due to acquired thrombophilia for the presence of APS. ${ }^{1}$ LAC is the more common acquired clotting inhibitor in APS and it is able to alter INR (besides aPTT). ${ }^{10,11}$ However, the literature suggests that this kind of interference may appear more frequently if INR value is higher than $4.0 .^{4}$

Our data confirm that patients ongoing OA for a thrombotic event without LAC positivity usually have good control of INR with a common reagent (ie, RecombiPlasTin $2 \mathrm{G}$, as thromboplastin); yet, those with LAC positivity show less control of INR values because of the interference of LAC. However, this aspect seems to be relevant for INR values greater than 4 , while values less than 4 did not determine significant alteration in the laboratory assays. Subjects with LAC positivity that show INR values greater than 4 during OA may benefit from a matched INR control with another

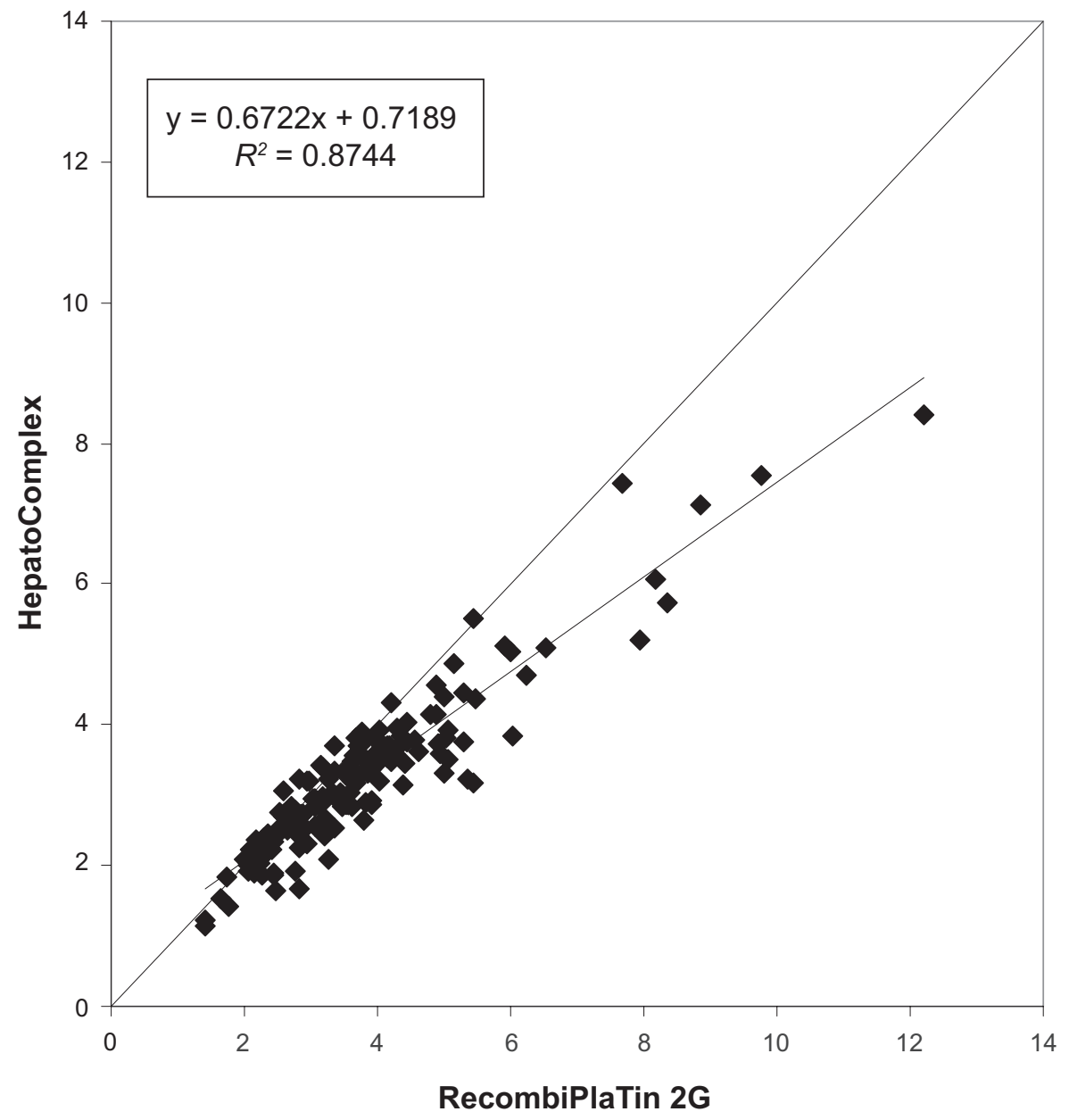

Figure I Cases vs controls linear regression of INR monitoring with or without lupus anticoagulant positivity with two different thromboplastins. 
thromboplastin (ie, HepatoComplex) that has less sensibility to acquired clotting inhibitors as LAC. Alternatively for these values it may suggested to perform assays for Xa levels in order to exclude other types of interferences in the management of antithrombotic therapies. ${ }^{8}$

So, two different INR values due to the interference of an acquired inhibitor as LAC may also alter the daily clinical approach if we need to choose appropriate doses of AVK drugs for long-term OA. From a clinical point of view, we should consider that this kind of patient might go on a more frequent control of INR during OA in order to avoid mistakes in chronic long term therapy, although our data did not underline this aspect. This approach, using another thromboplastin less sensitive to acquired inhibitor as LAC, permitted us to have good control of OA in the clinical setting avoiding thrombotic and hemorragic complications. We should consider, in fact, that a misunderstanding in the administered AVK drugs' doses may reflect a possible increased trend to develop thrombotic and/or hemorragic complications, which are the most common complications for patients ongoing long term treatment with AVK drugs.

In conclusion, because the possible interference of acquired clotting inhibitors, such as LAC, in the INR monitoring for OA, we suggest monitoring INR with a less sensitive thromboplastin such as HepatoComplex to avoid thrombotic and hemorragic complications. In particular, if the INR value is greater than 4 as reported by our data, a different approach in the administration of AVK drug is required.

\section{Disclosure}

The authors report no conflicts of interest in this work.

\section{References}

1. Büller HR, Agnelli G, Hull RD, Hyers TM, Prins MH, Raskob GE. Antithrombotic therapy for venous thromboembolic disease: the Seventh ACCP Conference on Antithrombotic and Thrombolytic Therapy. Chest. 2004;126(S3):401S-428S.

2. Perkins HA, Biben RL. Coagulation factor analysis in patients on longterm anticoagulation. Calif Med. 1970;112(3):1-7.

3. van den Besselaar AM. International standardization of laboratory control of oral anticoagulanttherapy: a survey of thromboplastin reagents used for prothrombin time testing. J Heart Valve Dis. 1993;2:42-52.

4. WHO Expert Committee on Biological Standardization. Guidelines for thromboplastins and plasma used to monitor oral anticoagulant therapy. World Health Organization Technical Report Series 1999; nr 889, Geneva, Switzerland: World Health Organization; 1999.

5. Della Valle P, Crippa L, Safa O, et al. Potential failure of the International Normalized Ratio (INR) system in the monitoring of oral anticoagulation in patients with lupus anticoagulants. Ann Med Interne. 1996;147:10-14.

6. Rapaport SI, Le DT. Thrombosis in the antiphospholipid antibody syndrome. N Engl J Med. 1995;333:665.

7. Moll S, Ortel TL. Monitoring warfarin therapy in patients with lupus anticoagulants. Ann Intern Med. 1997;127:177-185.

8. Tripodi A, Chantarangkul V, Clerici M, Negri B, Galli M, Mannucci PM. Laboratory control of oral anticoagulant treatment by the INR system in patients with antiphospholipid syndrome and lupus anticoagulant. Results of a collaborative study involving nine commercial thromboplastin. BJH. 2001;115:672-678.

9. Miyakis S, Lockshin MD, Atsumi T, Branch DW, Brey RL, Cervera $\mathrm{R}$, et al. International consensus statement on an update of the classification criteria for definite antiphospholipid syndrome (APS). J Thromb Haemost. 2006;4:295-306.

10. Brandt JT, Triplett DA, Alving B, Scharrer I. Criteria for the diagnosis of lupus anticoagulants: an update. Thromb Haemost. 1995;74:1185-1190.

11. Greaves M, Cohen H, Machin SJ, Mackie I. Guidelines on the investigation and management of the antiphospholipid syndrome. Br J Haematol. 2000;109:704-715.
Journal of Blood Medicine

\section{Publish your work in this journal}

The Journal of Blood Medicine is an international, peer-reviewed, open access, online journal publishing laboratory, experimental and clinical aspects of all topics pertaining to blood based medicine including but not limited to: Transfusion Medicine; Blood collection, Donor issues, Transmittable diseases, and Blood banking logistics; Immunohematology; Artificial and alternative

\section{Dovepress}

blood based therapeutics; Hematology; Biotechnology/nanotechnology of blood related medicine; Legal aspects of blood medicine; Historical perspectives. The manuscript management system is completely online and includes a very quick and fair peer-review system. Visit http://www.dovepress.com/ testimonials.php to read real quotes from published authors. 